\title{
Are rotating strange quark stars good sources of gravitational waves?
}

\author{
D. Gondek-Rosińska ${ }^{1,2}$, E. Gourgoulhon ${ }^{1}$, and P. Haensel ${ }^{2,1}$ \\ ${ }^{1}$ Laboratoire de l'Univers et de ses Théories, UMR 8102 du CNRS, Observatoire de Paris, 92195 Meudon Cedex, France \\ 2 Nicolaus Copernicus Astronomical Center, Bartycka 18, 00-716 Warszawa, Poland
}

Received 30 September 2002 / Accepted 25 July 2003

\begin{abstract}
We study the viscosity driven bar mode (Jacobi-like) instability of rapidly rotating quark matter stars (strange stars) in general relativity. A triaxial, "bar shaped" compact star could be an efficient source of continuous wave gravitational radiation in the frequency range of the forthcoming interferometric detectors. We locate the secular instability point along several constant baryon mass sequences of uniformly rotating strange stars described by the MIT bag model. Contrary to neutron stars, strange stars with $T /|W|$ (the ratio of the rotational kinetic energy to the absolute value of the gravitational potential energy) much lower than the corresponding value for the mass-shed limit can be secularly unstable to bar mode formation if shear viscosity is high enough to damp out any deviation from uniform rotation. The instability develops for a broad range of gravitational masses and rotational frequencies of strange quark stars. It imposes strong constraints on the lower limit of the frequency at the innermost stable circular orbit around rapidly rotating strange stars. The above results are robust for all linear self-bound equations of state assuming the growth time of the instability is faster than the damping timescale. Whether the instability can grow or not depends on many different physical quantities (e.g. value of viscosities (rather uncertain)). We discuss astrophysical scenarios where triaxial instabilities ( $r$-mode and viscosity driven instability) could be relevant (a new born star, an old star spinning up by accretion) in strange stars described by the standard MIT bag model of normal quark matter. The spin evolution of a strange star strongly depends on the strange quark mass. Taking into account actual values of viscosities in strange quark matter and neglecting the magnetic field we show that Jacobi-like instability cannot develop in any astrophysicaly relevant temperature windows. The main result is that strange quark stars described by the MIT bag model can be accelerated to very high frequency in Low Mass X-ray binaries if the strange quark mass is consistent with values based on particle data tables.
\end{abstract}

Key words. dense matter - equation of state - gravitation - relativity - stars: neutron - stars: rotation

\section{Introduction}

The first generation laser interferometric gravitational wave detectors such as LIGO, VIRGO and GEO600 should be fully operationally soon and second generation detectors like LIGO II within a few years. Triaxial instabilities of rotating compact stars (neutron stars, strange quark stars) can play an important role as emission mechanisms of gravitational waves in the frequency range of these detectors (see e.g. Stergioulas 1998, 2003 or Andersson 2003 for a review). A rapidly rotating relativistic star can spontaneously break its axial symmetry if the ratio of the rotational kinetic energy to the absolute value of the gravitational potential energy $T /|W|$ exceeds some critical value (Gondek-Rosińska \& Gourgoulhon 2002; Di Girolamo \& Vietri 2002; Shapiro \& Zane 1998; Bonazzola et al. 1996, 1998). The $m=2, l=2$ "bar mode" instability is the fastestgrowing instability if a rotation rate is sufficiently high.

Send offprint requests to: $\mathrm{D}$. Gondek-Rosińska,

e-mail: Dorota.Gondek@obspm.fr
Neutron stars can be forced to rotate rigidly if viscosity is high enough to damp out any deviation from uniform rotation. The viscosity-driven bar mode (Jacobi-like) instability of neutron stars has been studied in general relativity by Bonazzola et al. (1996, 1998), who find that only the stiffest equations of state (EOS) allow symmetry breaking. Since for "realistic" EOS of neutron stars the ratio of $T /|W|$ has a low value (usually lower than 0.14 ), this instability takes place only when a star is rotating at a frequency close to the mass-shed limit and has high mass.

Strange quark stars (SS) are currently considered as a possible alternative to neutron stars as compact objects (see e.g. Weber 1999 for a review). Claims of discovery have been recently announced (Drake et al. 2002), but the evidence is not yet decisive (Walter \& Lattimer 2002). It has been suggested by Gourgoulhon et al. (1999), Gondek-Rosińska et al. (2000a,b; 2001a) that a triaxial instability could develop more easily in a uniformly rotating strange star than in a neutron star. It was shown that independent of any details of the EOS of strange matter, the ratio $T /|W|$ for self-bound objects like strange stars 
can be even more than twice as high as for any models of neutron stars. No stability analysis in general relativity has been performed for strange stars untill now, because numerical constraints prevented investigators from treating stars with strongly discontinuous density profiles at the surface. In the present paper we perform a numerical study of the secular triaxial instability of rigidly rotating strange stars in general relativity. We use the code developed recently by Gondek-Rosińska \& Gourgoulhon (2002) for locating the secular bar mode instability points of uniformly rotating incompressible fluid bodies in general relativity. Numerous tests have been performed to assess the validity of the method and the accuracy of the numerical code (Gondek-Rosińska \& Gourgoulhon 2002).

In this paper we shall focus on the following questions: for which gravitational masses and rotational frequencies of strange stars does the Jacobi-like instability occur? Does gravitational radiation set strong limits on spin rate of strange stars and on the frequency at the innermost stable circular orbit around rapidly rotating strange stars? What astrophysical scenarios does the viscosity driven instability allow one to develop? How do our conclusions depend on physical parameters of the MIT bag model?

The plan of the paper is as follows. The description of previous studies of the bar mode instabilities is presented in Sect. 2. Sections 3 and 4 are devoted to the description of the strange matter EOS used by us and of the numerical technique we employed, respectively. The numerical results and discussion are presented in two sections (Sects. 5 and 6). In Sect. 5 we examine the necessary condition for the onset of the instability: that the frequency of a corotating mode goes through zero in a frame co-rotating with the star. This condition yields the critical angular velocity (at a given compactness) for which the instability could start to operate, provided it is not damped by other mechanisms. The results are robust for any linear selfbound EOS. In Sect. 6. we discuss the astrophysical scenarios when triaxial instabilities could be relevant. This part includes an estimate of different timescales. Finally, Sect. 7 provides a summary of our work.

\section{Previous studies of the bar mode instability}

Rapidly rotating compact stars may be subject to different kinds of bar mode non-axisymmetric rotational instabilities.

There exist two different classes and corresponding time-scales for non-axisymmetric instabilities: a dynamical instability, growing on the hydrodynamical time-scale and secular instabilities which grow on much longer dissipation time-scales. In Newtonian theory, a rapidly rotating incompressible fluid body (a Maclaurin spheroid) is secularly unstable to a bar-mode formation, if $T /|W|>0.1375$ (Jacobi/Dedekind bifurcation point) and dynamically unstable if $T /|W|>0.2738$ (Chandrasekhar 1969). A secular instability can grow only in the presence of dissipative mechanisms, such as shear viscosity (Roberts \& Stewartson 1963) or gravitational radiation (the Chandrasekhar-Friedman-Schutz instability (hereafter CFS instability; Chandrasekhar 1970; Friedman \& Schutz 1978; Friedman 1978). Viscosity dissipates mechanical energy but preserves angular momentum. As shown by
Christodoulou et al. (1995), the viscosity driven (Jacobi-like) bar mode instability appears only if the fluid circulation is not conserved. If on the contrary, the circulation is conserved (as in inviscid fluids submitted only to potential forces), but not the angular momentum, it is the gravitational radiation (Dedekind-like) instability which develops instead. If both viscosity and gravitational radiation are operative, they act against each other. Which of the two dissipative mechanisms dominates is highly dependent on the viscosities and the temperature of a star.

The few numerical studies of secular instabilities in rapidly rotating stars in general relativity show that nonaxisymmetric modes driven unstable by viscosity no longer coincide with those made unstable by gravitational radiation. As was shown by recent post-Newtonian (Shapiro \& Zane 1998; Di Girolamo \& Vietri 2002) and relativistic (Gondek-Rosińska \& Gourgoulhon 2002) studies of viscosity-driven instability in rigidly rotating incompressible fluid bodies, general relativity weakens this bar mode instability: the values of $T /|W|$, eccentricity and rotation rate increase at the onset of instability as the compaction parameter $G M /\left(R_{\mathrm{circ}} c^{2}\right)$ increases. This stabilizing tendency of general relativity is in agreement with previous studies of Jacobi-like instability of rigidly rotating compressible (neutron) stars (Bonazzola et al. 1996, 1998). The stabilizing effect of relativity is not very strong, the critical value of $T /|W|$ for relativistic neutron star models is 0.14 (the Newtonian value is 0.1275 (Bonazzola et al. 1996) for compressible bodies) and 0.18 for very relativistic (with proper compaction parameter $M / R=0.25$ ) incompressible fluid body.

On the other hand, the gravitational-radiation driven instability is strengthened by relativity. As was first proved by Friedman \& Schutz (1978) the Dedekind-like instability is generic in rotating stars - one can always find a mode that is unstable for a star with a given rotation rate. The most important pulsation modes for the CFS instability are the $r$-mode and $f$-mode (Stergioulas 1998, 2003; Andersson 2003; Andersson \& Kokkotas 2001; Stergioulas \& Friedman 1998; Friedman \& Lockitch 2002, for recent reviews of these instabilities). For a wide range of realistic EOS, Morsink et al. (1999) find that the critical $T /|W|$ for the onset of the instability in the $l=2$ $f$-mode is only $T /|W| \sim 0.08$ for models with mass $1.4 M_{\odot}$ and $T /|W| \sim 0.06$ for maximum mass models. However, based on the relative strength of the $f$-mode and $r$-mode instabilities for NS, one can expect that the $r$-mode instability will occur at lower rotation rates and in a larger temperature window than the $f$-mode instability, unless some damping mechanism favors a stronger damping at the $r$-mode frequency. The onset of the $r$-mode instability for SS has been studied by Madsen (2000) and Andersson et al. (2002).

The dynamical instability against bar-mode deformation of rapidly rotating polytropic stars was studied by Shibata et al. (2000) in full general relativity and by Saijo et al. (2001) in the first post-Newtonian approximation. The authors considered differentially rotating proto-neutron stars. Their main result is that in general relativity the onset of dynamical instability occurs for a somewhat smaller critical value of $(T /|W|)_{\text {crit }} \sim 0.24-0.25$ than the Newtonian value $\sim 0.27$ for incompressible Maclaurin spheroids. 


\section{Equation of state}

The possibility of the existence of quark matter dates back to the early seventies. Bodmer (1971) remarked that matter consisting of deconfined up, down and strange quarks could be the absolute ground state of matter at zero pressure and temperature. If this is true then objects made of such matter, so-called "strange stars", could exist (Witten 1984). Typically, strange stars are modeled with an equation of state based on the phenomenological MIT-bag model of quark matter (Alcock et al. 1986; Haensel et al. 1986), in which quark confinement is described by an energy term proportional to the volume (Farhi \& Jaffe 1984).

In the framework of this model the quark matter is composed of massless $\mathrm{u}, \mathrm{d}$ quarks, massive s quarks and electrons. There are three physical quantities entering the model: the mass of the strange quarks, $m_{\mathrm{s}}$, the bag constant, $B$, and the strength of the QCD coupling constant $\alpha$.

The EOS of strange quark matter (SQM) is calculated using a perturbation expansion in $\alpha$. The expansion is treated in the renormalization scheme. In particular, one has to use renormalized values of $m_{\mathrm{s}}$ and $\alpha$, which both depend on the selected value of the renormalization point, $\rho_{\mathrm{R}}$ (see, e.g., Farhi \& Jaffe 1984). The value of $\rho_{\mathrm{R}}$ is of the order of typical energies in the quark-system under consideration. Following Farhi \& Jaffe (1984), we will use $\rho_{\mathrm{R}}=313 \mathrm{MeV}$.

\subsection{The strange quark mass}

The s quark mass given in the tables compiled by the Particle Group is the so called current quark mass, $m_{\mathrm{s}}^{(\mathrm{c})}=75-150 \mathrm{MeV}$ (Groom et al. 2000). Such a mass can be used in the systems in which the energies of the relative quark-quark motion $\gg 1 \mathrm{GeV}$, say $100 \mathrm{GeV}$, which is many orders of magnitude larger than typical energies in SQM. Therefore, the value of $m_{\mathrm{s}}$ has to renormalized using the renormalization group equation, and its value calculated at $\rho_{\mathrm{R}}$ (Farhi \& Jaffe 1984). The lowest-order (in $\alpha$ ) renormalization group equation for $m_{\mathrm{s}}$ indicates that renormalization will significantly increase the s-quark mass, and that with our choice of $\rho_{\mathrm{R}}$ we will have $m_{\mathrm{s}} \sim 2 m_{\mathrm{s}}^{(\mathrm{c})}$. In what follows, we will use $m_{\mathrm{s}} c^{2}=200 \mathrm{MeV}$ as a basic (standard) value of $m_{\mathrm{s}}$ in SQM, but we will also present some results for $m_{\mathrm{s}}=100 \mathrm{MeV}$, to visualize sensitivity of our results to the value of $m_{\mathrm{s}}$.

\subsection{Models of EOSs of strange matter}

Our basic EOS corresponds to the standard values of the MIT bag model parameters of SQM:

- SQS1 - the standard MIT bag model: $m_{\mathrm{s}} c^{2}=200 \mathrm{MeV}$, $\alpha=0.2, B=56 \mathrm{MeV} / \mathrm{fm}^{3}$;

We shall also find the onset of the viscosity driven instability for two other MIT bag models to check how our results depend on model parameters:

- SQS0 - the simplified MIT bag model: $m_{\mathrm{s}}=0, \alpha=0$; $B=60 \mathrm{MeV} / \mathrm{fm}^{3}$;
- SQS2 - the "extreme" MIT bag model (relatively low strange quark mass and $B$ but very high $\alpha$ ): $m_{\mathrm{s}} c^{2}=$ $100 \mathrm{MeV}, \alpha=0.6, B=40 \mathrm{MeV} / \mathrm{fm}^{3}$.

\section{Numerical codes}

\subsection{Axisymmetric equilibrium stars}

We have calculated axisymmetric models of rotating strange stars using two different highly accurate relativistic codes. The first code developed by Gourgoulhon et al. (1999) is based on the multi-domain spectral method of Bonazzola et al. (1998). This code has been used previously for calculating properties of rapidly rotating strange stars described by the MIT bag model (Gourgoulhon et al. 1999; Gondek-Rosińska et al. 2000a, 2001a; Zdunik et al. 2000a) and by the Dey et al. (1998) model of strange matter (Gondek-Rosińska et al. 2000b, 2001). To compute very rapidly rotating axisymmetric stationary configurations (when the ratio of polar to equatorial radius is lower than $0.3-0.5$ depending on the compaction parameter - see Sect. III.A of Gondek-Rosińska \& Gourgoulhon 2002 for a discussion), where the first code has difficulties converging to a solution, we employ a second numerical code (Stergioulas \& Friedman 1995, see Stergioulas 1998 for a description). In this code the equilibrium models are obtained following the KEH method (Komatsu et al. 1989), in which the field equations are converted to integral equations using appropriate Green functions. Models computed by both codes agree very well in all computed properties (Stergioulas et al. 1999).

\subsection{The secular bar mode instability}

Recently Gondek-Rosińska \& Gourgoulhon (2002) used multidomain method for calculating Jacobi-like instability of relativistic incompressible fluid bodies. They have introduced surface-fitted coordinates, which enable them to treat the strongly discontinuous density profile at the surface of incompressible bodies. This avoids any Gibbs-like phenomenon and results in a very high precision, as demonstrated by comparison with the analytical result for the Newtonian Maclaurin-Jacobi bifurcation point, that their code has retrieved with a relative error of $10^{-6}$. Their results are in close agreement with the very recent post-Newtonian calculations of viscosity driven bar mode instability in rigidly rotating incompressible fluid bodies by Di Girolamo \& Vietri (2002) (the relative difference is less than $1 \%$.)

The method of finding the secular bar mode instability point presented there is an improvement on an earlier method by Bonazzola et al. (1996, 1998). The approach consists of perturbing an axisymmetric and stationary configuration and studying its evolution by constructing a series of triaxial quasiequilibrium configurations. If the perturbation is damped (resp. grows) during the interation, the equilibrium configuration is declared to be stable (resp. unstable) (see Gondek-Rosińska \& Gourgoulhon 2002 for a description). The above method does not require us to specify some value of viscosity. Whatever this value, the effect of viscosity is simulated by the rigid rotation 
profile that is imposed at each step in the iterative procedure. If one considers the iteration as mimicking some time evolution, this means that the time elapsed between two successive steps has been long enough for the actual viscosity to have rigidified the fluid flow. Consequently, using this method one cannot get the instability time scale, which depends upon the actual value of the viscosity, but only the instability.

We use the same code as Gondek-Rosińska \& Gourgoulhon (2002) for finding the viscosity driven instability points of rapidly rotating strange stars described by the MIT bag model.

\section{Calculations and results}

\subsection{Effect of relativity on the secular bar mode instability}

In this section we are focusing on the effect of relativity on the Jacobi-like instability in the case of strange quark stars. Strange stars in contrast to ordinary NS have a stiff EOS at the surface and are soft inside and their density profiles are flat. Rotating strange stars with small masses, lower than $0.1 M_{\odot}$, can be described very well by a uniform-density fluid, i.e., the classical Maclaurin spheroids (see Amsterdamski et al. 2002 for comparison between fully relativistic calculations of rapidly rotating low mass strange stars and Maclaurin spheroids). Widely used indicators for the onset of viscosity driven instability are the ratio of the kinetic energy to the absolute value of the gravitational potential energy $T /|W|$ and the eccentricity $e=\left(1-\left(r_{\mathrm{p}} / r_{\mathrm{eq}}\right)^{2}\right)^{1 / 2}$ where $r_{\mathrm{p}}$ and $r_{\mathrm{eq}}$ are the polar and equatorial coordinate radius. We have computed the $T /|W|$ according to the prescription of Friedman et al. (1986):

$$
T /|W|=\frac{\Omega J / 2}{\Omega J / 2+M_{\mathrm{p}}-M}
$$

where $\Omega$ is the angular velocity, $J$ the stellar angular momentum, $M$ is the gravitational mass and $M_{\mathrm{p}}$ is the "proper" internal energy. A rotating configuration becomes unstable to a bar-mode formation if the ratio of $T /|W|$ or the eccentricity exceed some critical value. For incompressible Newtonian bodies, the critical values of $T /|W|$ and $e$ are 0.1375 and 0.8126 respectively. The Newtonian value of $T /|W|$ is 0.1275 (Bonazzola et al. 1996) for compressible bodies.

General relativity weakens the viscosity driven bar mode instability, but the stabilizing effect is not very strong (Gondek-Rosińska \& Gourgoulhon 2002; Di Girolamo \& Vietri 2002; Bonazzola et al. 1996, 1998). The ratio $T /|W|$ for relativistic neutron star models is $\sim 0.14$ and for highly relativistic $\left(G M /\left(R_{\text {circ }} c^{2}\right)=0.25\right)$ uniformly rotating incompressible bodies is only $30 \%$ higher than the Newtonian value (Gondek-Rosińska \& Gourgoulhon 2002). It was found by Gondek-Rosińska \& Gourgoulhon (2002) that for rigidly rotating homogeneous fluid bodies the dependence of $(T /|W|)_{\text {crit }}$ on the proper compactness parameter $x:=G M /\left(R_{\text {circ }} c^{2}\right)$ can be very well approximated by the function

$$
(T /|W|)_{\text {crit }}=(T /|W|)_{\text {crit,Newt }}+0.148 x(x+1) .
$$

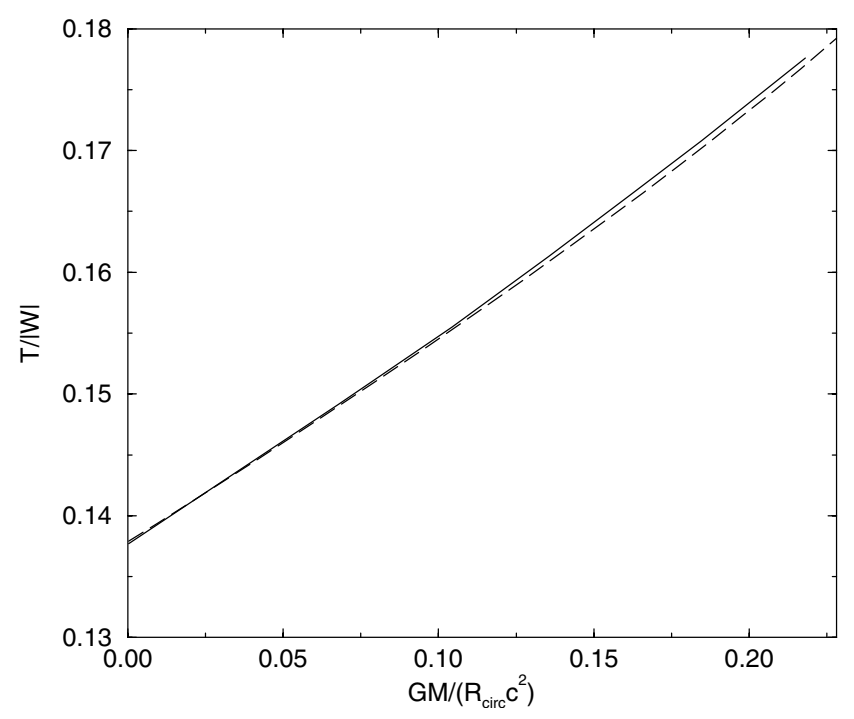

Fig. 1. Effect of general relativity on the critical value of the kinetic energy to the absolute value of the gravitational potential energy for different models of strange quark stars described by MIT bag model (solid line) and incompressible fluid stars calculated by Gondek-Rosińska \& Gourgoulhon (2002) (dashed line). The ratio of $T /|W|$ for compaction parameter $G M /\left(R_{\text {circ }} c^{2}\right)=0$ corresponds to the classical Newtonian Jacobi/Dedekind bifurcation point, 0.1375 .

We locate the onset of instability (see Sect. 4.2 for description of our method) along several constant baryon mass sequences of uniformly rotating axisymmetric strange quark stars described by different MIT bag models (see Sect. 3.) for the compaction parameter $G M /\left(R_{\text {circ }} c^{2}\right)=0-0.25$. To show the role of relativistic effects we plot in Fig. 1 the ratio of $T /|W|$ as a function of the proper compaction parameter at the secular instability points for strange stars. All 3 models described in Sect. 3 are represented by a solid line. We compare our results with those obtained by Gondek-Rosińska \& Gourgoulhon (2002) for relativistic incompressible bodies (dashed line). We see the same dependence of the critical value of $(T /|W|)_{\text {crit }}$ on compactness in both cases. In the Newtonian limit $\left(G M /\left(R_{\text {circ }} c^{2}\right)=0\right)$ the ratio of $(T /|W|)_{\text {crit }}$ is 0.1375 .

In Fig. 2 we plot the oblateness of the star (the ratio of $\left.r_{\mathrm{p}} / r_{\mathrm{eq}}\right)$ versus the proper compaction parameter for both MIT bag model strange stars (solid line) and incompressible fluid bodies (dashed line). It was found by Gondek-Rosińska \& Gourgoulhon (2002) that the critical value of the eccentricity depends very weakly on the degree of relativity and for $G M /\left(R_{\text {circ }} c^{2}\right)=0.25$ is only $2 \%$ larger than the Newtonian value at the onset for the secular bar mode instability. In the case of strange stars the dependence is a little bit stronger. For massive strange stars the density profile is no longer completely flat - for example the ratio of central density to surface density for a strange star is 1.25 and 1.8 for proper compaction parameters 0.1 and 0.2 respectively.

\subsection{Results for the standard MIT bag model}

Here we present results for the standard MIT bag model, SQS1, where the mass of strange quark is taken to be $200 \mathrm{MeV} / \mathrm{c}^{2}$, the 


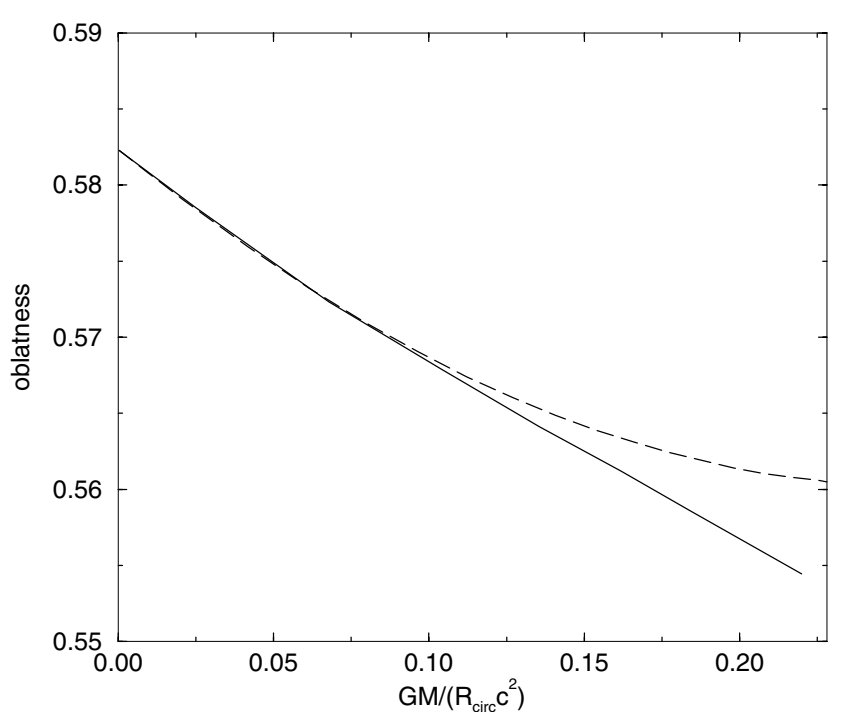

Fig. 2. Critical values of stellar oblateness as a function of the proper compaction parameter for different models of strange quark stars described by MIT bag model (solid line) and incompressible fluid stars (dashed line).

coupling constant 0.2 and the bag constant $B=56 \mathrm{MeV} / \mathrm{fm}^{3}$. We show that the results presented in this section can be applied to the other MIT bag models of SS to a very good approximation (Sect. 5.3).

\subsubsection{Constant baryon mass sequences}

We construct constant baryon mass sequences of rotating strange stars described by the SQS1 model, i.e. the so-called evolutionary sequences. This is relevant for the evolution of an isolated strange star slowly losing its energy and angular momentum via gravitational and electromagnetic radiation. In general we cannot neglect accretion on a compact star (e.g. an accretion from supernova on a just born strange star or from a companion in low mass X-ray binaries).

We compute both normal (with $M_{\mathrm{b}}=0.8,1.6,2.1 M_{\odot}$ ) and supramassive sequences $\left(2.5,3.0 M_{\odot}\right)$. A sequence is called normal if it terminates at the zero angular momentum limit with a static, spherically symmetric solution, and it is called a supramassive sequence if it does not. The boundary between these two sequences is the sequence with the maximum baryon mass of a static configuration, which is $2.17 M_{\odot}$ for the SQS1 model. We consider configurations stable with respect to axisymmetric perturbations, i.e. for a supramassive constant baryon mass sequence a model is stable to quasi-radial perturbations, if $\left(\frac{\partial J}{\partial \rho_{\mathrm{c}}}\right)_{M_{\mathrm{b}}}<0\left(\right.$ or $\left.\left(\frac{\partial M}{\partial \rho_{\mathrm{c}}}\right)_{M_{\mathrm{b}}}<0\right)$ where $M_{\mathrm{b}}$ is the baryon mass of the star and $\rho_{\mathrm{c}}$ is the central density of the star (Friedman et al. 1988).

The dependence of angular momentum versus central density as well versus rotational frequency for the constant baryon sequences is shown in Fig. 3. Each sequence is represented by a solid line and is labeled by the baryon mass as well as by the gravitational mass of the static configuration (in parentheses), if it exists. The gravitational mass of a strange star rotating at the Keplerian limit is only a few percent (up to $7 \%$ ) higher than $M$ of the static member of the sequence (for the influence of rotation on the gravitational mass see Figs. 2 in Gondek-Rosińska et al. 2000b; Gondek-Rosińska et al. 2001a). Marginally stable configurations to quasi-radial perturbation are shown as a dot-dashed line. The angular momentum increases along each sequence from $J=0$ for static configurations, or $J_{\min }$ on the dash-dotted line for supramassive stars, to $J_{\max }$ for the Keplerian configurations (thin short dashed line, as well as the crosses for each considered sequence). The star in a sequence reaches the mass-shedding limit if the velocity at the equator of a rotating star is equal to the velocity of an orbiting particle at the surface of the star. It is worth noting (Fig. 3) that for any given baryon mass, the maximal rotating configuration is not mass-shedding one. The mass-shed configurations are reached due to the increase of the equatorial radius relative to the deformation of the rotating star. Such a phenomenon was discussed by Zdunik et al. (2000b) in the case of normal sequences of MIT bag model strange stars and Gondek-Rosińska et al. (2000) for the Dey et al. (1998) EOS. This feature is characteristic of stars described by a self-bound linear EOS. If a strange star is born with high angular momentum (close to the maximum available) it will spin up, losing the angular momentum (e.g. by emitting of gravitational waves).

Marginally stable configurations to non-axisymmetric perturbation are shown as a long-dashed line (as well as a filled circle for each considered constant baryon mass sequence). As mentioned in Sect. 4, the multi-domain spectral technique with surface-fitted coordinates that we employ has some trouble when $r_{\mathrm{p}} / r_{\mathrm{eq}}<1 / 3-0.5$ depending on the compaction parameter. To compute very oblate and highly relativistic (with high compaction parameter) rotating axisymmetric stationary configurations we use the Stergioulas \& Friedman (1995) code, but we cannot locate the Jacobi-like bifurcation point in this case. The thin part of the long dashed line (for $f>1.3 \mathrm{kHz}$ for the SQS1 model) represents marginally stable configurations found in the following way - we noticed that the dependence of central enthalpy $H_{\mathrm{c}}$ on rotational frequency $f\left(H_{\mathrm{c}}\right.$ and $f$ are two input parameters needed for finding a rotating star described by given equation of state) at the onset of instability is very well approximated by the following formula: $H_{\mathrm{c}}=0.47 f_{[\mathrm{kHz}]}-0.45$ for SQS1 model. We extrapolated this formula to higher rotational frequency in order to find central enthalpy to compute the axisymmetric configuration.

Thick parts of the solid lines, between the filled circle and the cross, correspond to configurations secularly unstable to the bar mode formation. These configurations can be good emitters of gravitational waves. However a strange quark star described by this model must rotate very rapidly, with frequency higher than $1.1 \mathrm{kHz}$ for $1.4 M_{\odot}$, to allow symmetry breaking. If viscosity is high (see discussion in Sect. 6), an accreting strange star (belonging to a normal sequence) in low mass X-ray binaries (LMXBs) will never reach the mass-shedding limit. It can be accelerated to the critical frequency (which can be higher than the Keplerian frequency - see Fig. 3), corresponding to the marginally stable configuration to the bar mode formation instead. 

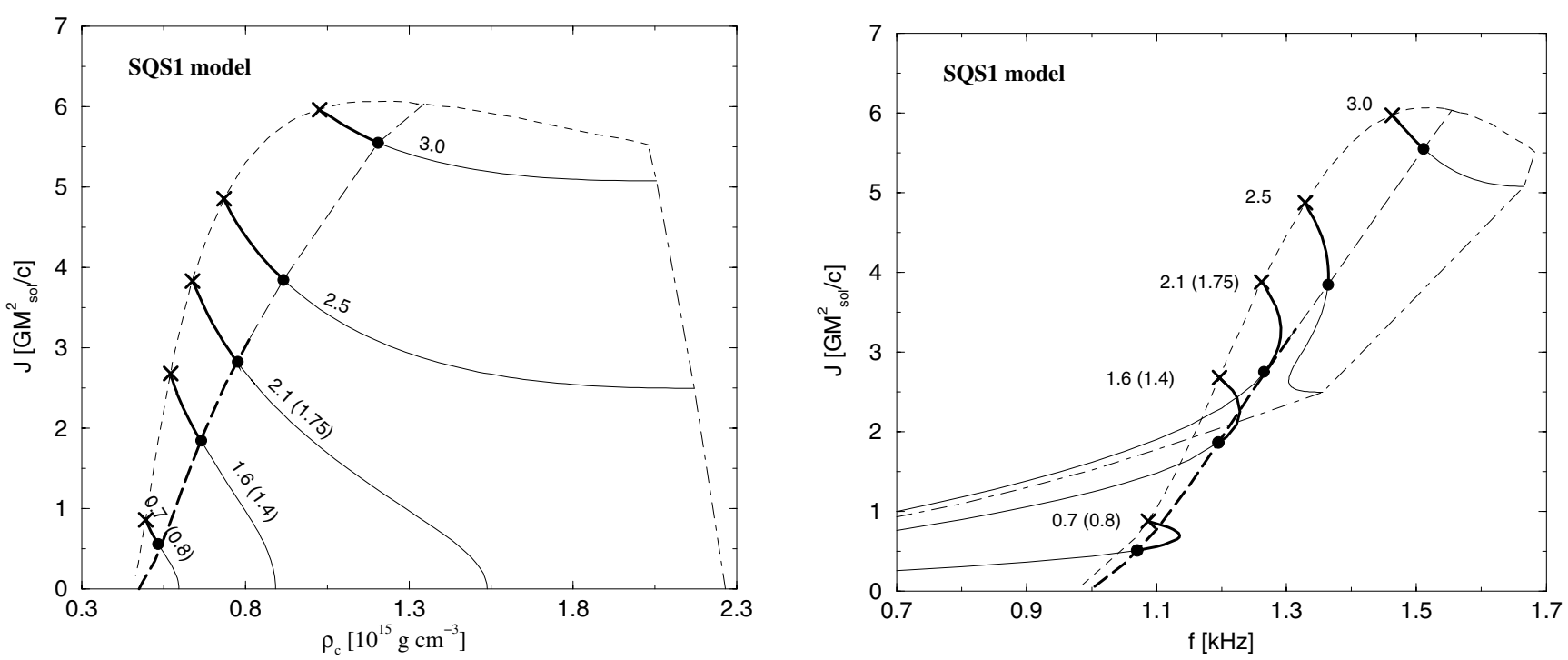

Fig. 3. Angular momentum as a function of central density (left) and the rotation frequency $f=\Omega /(2 \pi)$ (right) along sequences of constant baryon mass (solid lines). Each solid line is labeled by this baryon mass in solar mass units, as well as (in parentheses) by the gravitational mass of the static configuration, if it exists. The dash-dotted line indicates stars marginally stable to axisymmetric perturbations. The Keplerian configurations are shown as thin short-dashed line (also as crosses for each of considered sequences). The long dashed line indicates stars marginally stable to non-axisymmetric perturbations. Thick solid lines represent the configurations secularly unstable to the bar mode formation taking into account viscosity as a leading dissipative mechanism

\subsubsection{Masses and rotational frequencies for secularly unstable strange quark stars}

The crucial question from the observational point of view is - for which masses and frequencies the triaxial instability in the case of strange stars can develop. In order to answer this question we plot the value of $T /|W|$ versus gravitational mass for several constant frequency $f=\Omega / 2 \pi$ sequences (thin solid lines) of uniformly rotating strange stars in Fig. 4. On one end of each sequence there is a mass-shedding configuration (with the lowest central density in the sequence), and on the other end the last stable configuration with respect to axisymmetric perturbations (the densest object in the sequence). The dotted line separates normal strange stars from supramassive strange stars.

We see that the ratio of $T /|W|$ for strange quark stars rotating at the mass-shedding limit is very large and can be even higher than $T /|W|=0.32$. The $T /|W|$ is very high for all linear self-bound EOS (see Gondek-Rosińska et al. 2000b for different MIT bag models and Gondek-Rosińska et al. 2000a for strange stars described by the Dey model). The values of $T /|W|$ for strange stars are much larger than for any neutron star models (for which the maximum $T /|W|$ ranges typically from 0.1 to 0.14 - see e.g. Cook et al. 1994). In contrast to neutron stars, the $T /|W|$ for strange stars increases with decreasing stellar mass for all models. Very high values of $T /|W|$ are characteristic not only of supramassive stars but also of rapidly rotating strange stars with small and moderate masses. The large value of $T /|W|$ results from a flat density profile combined with strong equatorial flattening of rapidly rotating strange stars. The long-dashed line represents the onset of the secular bar mode instability (critical values of $T /|W|$ and $e$ ). Configurations

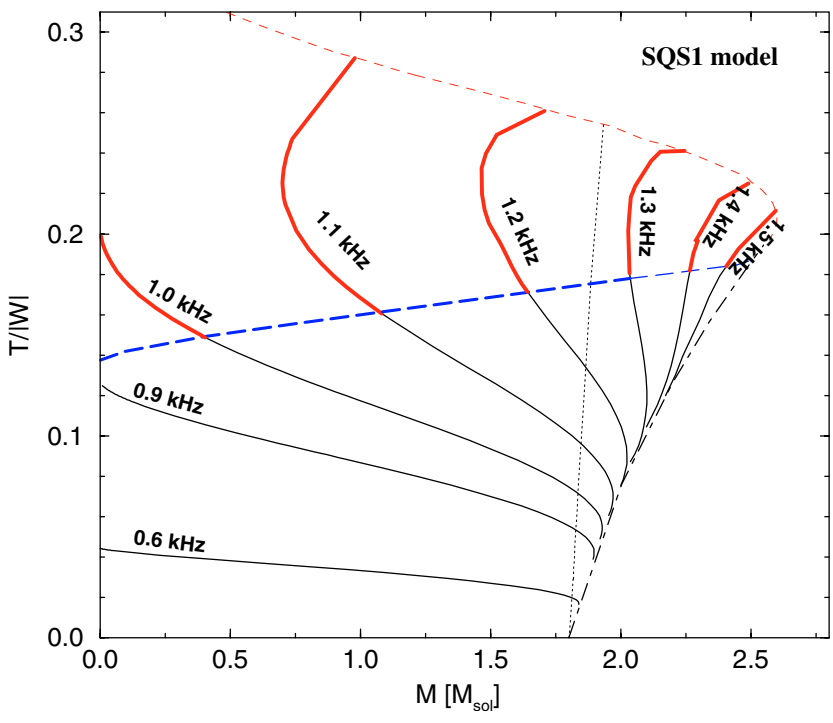

Fig. 4. The ratio of the kinetic energy to the absolute value of the gravitational potential energy as a function of gravitational mass for the standard MIT bag model of strange quark stars. The Newtonian limit is obtained for $M \rightarrow 0\left(J \rightarrow 0, \rho_{\mathrm{c}} \rightarrow \rho_{0}\right)$ (a rotating strange star can be considered as the Maclaurin spheroid). The solid lines correspond to sequences of configurations rotating with constant rotational frequency. The rotational frequency is labeled close to each line. On one end of each sequence there is a mass-shed configuration (shortdashed line) and on the other end the last stable configuration with respect to axisymmetric perturbations (dash-dotted line). The dotted line separates normal strange stars from supramassive strange stars. The meaning of the other lines is the same as in Fig. 3. Configurations with $T /|W|$ higher than represented by long-dashed line (e.g. thick parts of solid lines) are secularly unstable to the bar mode formation taking into account viscosity as a leading dissipative mechanism. 


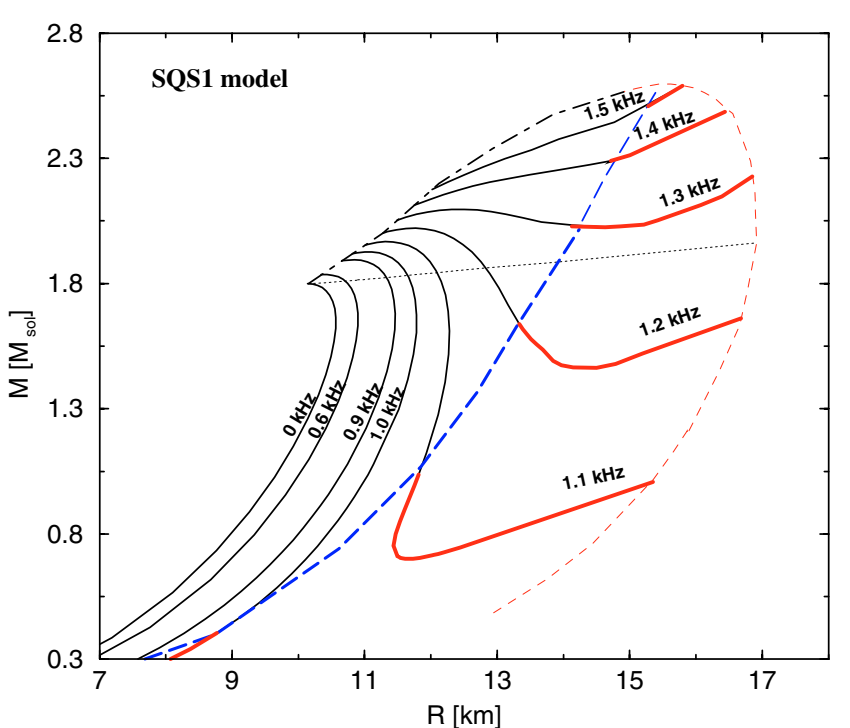

Fig. 5. Gravitational mass vs. radius for stars described by the standard MIT bag model. All lines have the same meaning as in Fig. 4. Models located to the right of the long dashed line are secularly unstable to the viscosity driven bar mode formation.

with $T /|W|$ higher than represented by this line are unstable with respect to non-axisymmetric perturbation and can be good emitters of gravitational waves (thick solid lines).

From Figs. 4 and 5 we see that strange stars could break their symmetry far away from the Keplerian limit and for broad ranges of masses and rotational frequencies. The critical value of $T /|W|$ is in the range $0.1375-0.18$ for very low mass rotating strange stars (in the Newtonian limit very well approximated by Maclaurin spheroids) and supramassive stars respectively. Those values are much lower than the Newtonian value of the dynamical instability against bar-mode deformation, 0.27 (according to our knowledge no stability analysis has been performed yet for uniformly rotating stars in general relativity). Existence of a crust only marginally affects our results. It has little influence on location of the viscosity driven instability points. According to Zdunik et al. (2001) the maximal values of $T /|W|$ at mass-shedding limit are only $20 \%$ lower for rapidly rotating strange stars with a maximal crust than the corresponding values for bare strange stars described by the same MIT bag model. From Fig. 3 we see that a high rotational frequency, higher than $0.95 \mathrm{kHz}\left(>1.1 \mathrm{kHz}\right.$ for $\left.1.4 M_{\odot}\right)$, is needed for a star described by the standard MIT bag model to reach the instability region. If we consider a model with $m_{\mathrm{s}}=200 \mathrm{MeV} / \mathrm{c}^{2}$, but with high coupling constants $\alpha=0.6$ and low bag constants $B=36.4 \mathrm{MeV} / \mathrm{fm}^{3}$ then the critical frequency for which the viscosity driven bar mode instability could occur is always $>0.77 \mathrm{kHz}$ and is $0.88 \mathrm{kHz}$ for a canonical strange star.

\subsection{Results for other MIT bag models}

In Fig. 6 we show gravitational masses and rotational frequency for which triaxial instabilities in rotating strange quark stars can develop. Each line corresponds to the onset of instability for particular MIT bag model described in Sect. 3. Strange

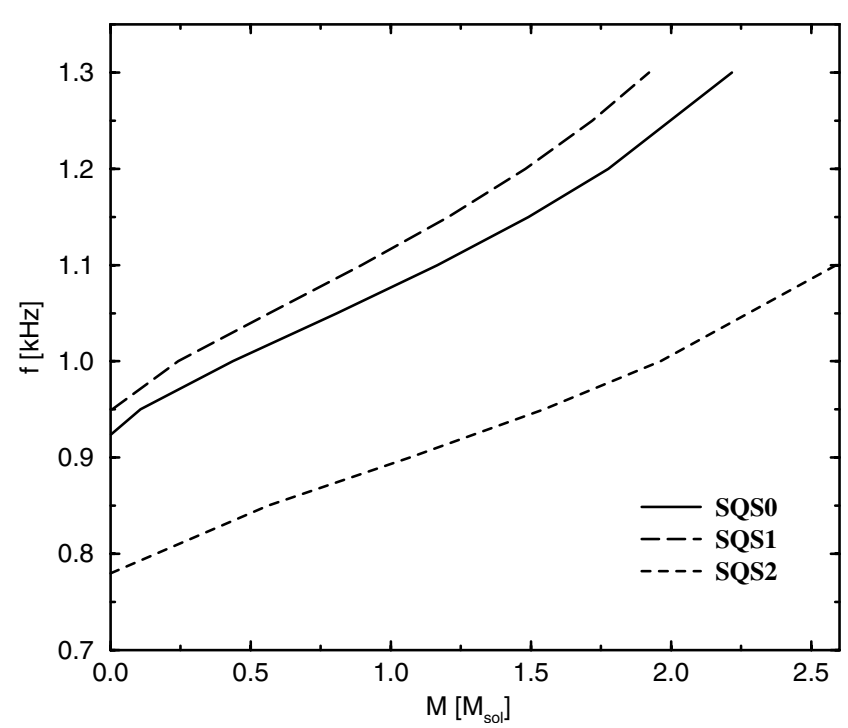

Fig. 6. Rotational frequency vs. gravitational mass for secular (viscosity driven) instability configurations described by different MIT bag models. Stars rotating with higher frequency than critical one (shown by each curve) are secularly unstable to non-axisymmetric perturbations.

stars rotating with a frequency higher than represented by this line are unstable with respect to non-axisymmetric perturbations and can be good emitters of gravitational waves. We see that viscosity driven instability develops for a broad range of gravitational masses of strange stars (from that of a planetoid to about three solar masses) independently of the model. However, a high rotational frequency (always higher than the frequency $\sim 0.64 \mathrm{kHz}$ of the fastest observed pulsar) is needed for a star to reach the instability region. The higher the gravitational mass the higher is the frequency needed to form a "barshaped star". The most comfortable situation is for MIT bag models with low MIT bag constant (e.g. SQS2) and for small stellar masses. However one should keep in mind that detailed simulations of supernovae do not predict remnant masses less than $1.2 M_{\odot}$ (Timmes et al. 1996).

It was shown that strange stars described by different models of strange matter can be very well approximated (with accuracy 1-2\%) by a linear function (Zdunik 2000; Gondek-Rosińska et al. 2000a)

$P=a\left(\rho-\rho_{0}\right) c^{2}$

where $P$ is the pressure, $\rho$ the mass-energy density, and $c$ the speed of light. Both $\rho_{0}$ and $a$ are functions of the physical constants $B, m_{\mathrm{s}}$ and $\alpha$ for the MIT bag model. In general, Eq. (1) corresponds to self-bound matter with density $\rho_{0}$ at zero pressure and with a fixed sound velocity $(\sqrt{a} c)$ at all pressures. For the MIT bag model the density of strange matter at zero pressure is in the range $\sim 3 \times 10^{14}-6.4 \times 10^{14} \mathrm{~g} \mathrm{~cm}^{-3}$ and $a$ between 0.289 (for a strange quark mass of $m_{\mathrm{s}} c^{2}=250 \mathrm{MeV}$ ) and $1 / 3$ (for massless quarks). For a fixed value of $a$, all stellar parameters are subject to scaling relations with appropriate powers of $\rho_{0}, f \propto \rho_{0}^{1 / 2}$, where $f$ denotes either of the frequencies (rotational or orbital), and $M, R \propto \rho_{0}^{-1 / 2}$ (see e.g Witten 1984; Zdunik 2000). The scaling law for stellar parameters with $a$ 


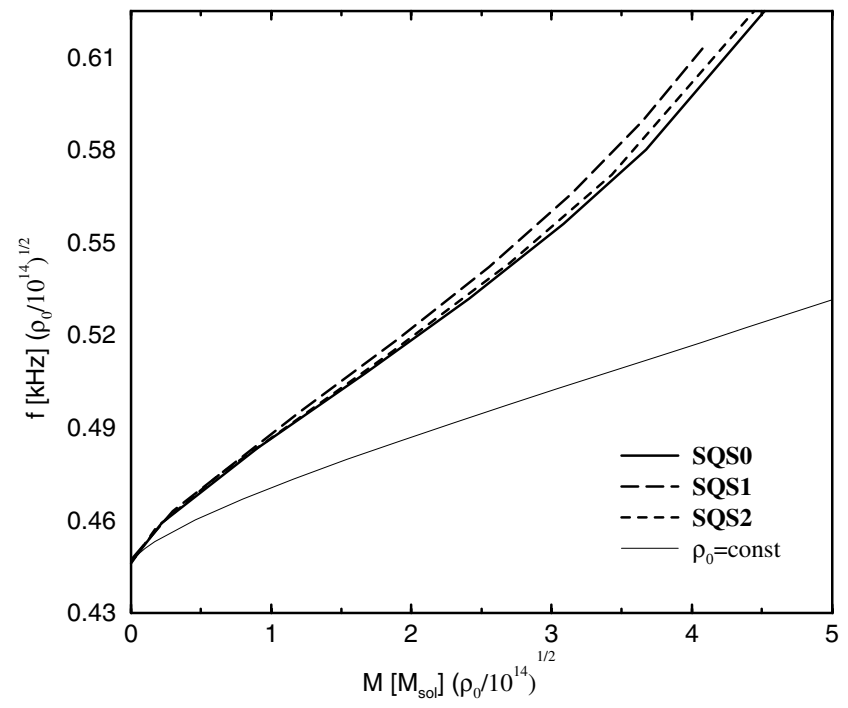

Fig. 7. Rescaled Fig. 6 to see how results depend on the $a$ parameter at equation of state given by Eq. (1). The onset points of stability for incompressible fluid bodies is shown for comparison (thin solid line). All other lines and symbols have the same meaning as in Fig. 6.

depends on rotational frequency (Gondek-Rosińska et al. 2004), for approximate scalings with $a$ at the limiting case of rotation at mass-shedding frequencies see Stergioulas et al. (1999).

In Fig. 7 the dependence of rotational frequency vs. mass for marginally stable strange stars to non-axisymmetric perturbations (Fig. 6) has been scaled out with appropriate powers of $\rho_{0}=10^{14} \mathrm{~g} \mathrm{~cm}^{-3}$. We see that the function $f(M)$ for onset points of instability very weakly depends on $a$ ( $a$ changes not very much in MIT bag model). The results obtained for one model can be used for other MIT bag models with the use of the scaling with $\rho_{0}$. The critical points for incompressible fluid stars with $\rho_{0}=$ const. $=10^{14}\left[\mathrm{~g} \mathrm{~cm}^{-3}\right]$ are shown for comparison (thin solid line).

From Fig. 7 we see that independently of any details of SS EOS, the lower the stellar mass the lower rotational frequency $f_{\text {crit }}$ needed to reach the instability region. Taking this into account we can find the absolute lower limit on rotational frequency of SS when viscosity driven instability can develop. In the Newtonian limit, when $M \rightarrow 0, J \rightarrow 0, \rho_{\mathrm{c}} \rightarrow \rho_{0}$, a rotating strange star can be considered as the Maclaurin spheroid for which the rotational frequency depends only on density and eccentricity (or $T /|W|$ ) (Chandrasekhar 1969). The square of the angular velocity at the onset point of viscosity driven instability $(e=0.8126)$ is

$\frac{\Omega_{\text {crit }}^{2}}{\pi G \rho_{0}}=0.3742$,

in other words for a strange star with gravitational mass $M \rightarrow 0$ the frequency $\frac{f_{\text {crit }}}{\sqrt{\rho_{0,14}}} \rightarrow 0.4458[\mathrm{kHz}]$. The lowest value of $f_{\text {crit }}$ is obtained for the lowest value of $\rho_{0}$ (fulfilling the condition of stability neutrons to spontaneous fusion into droplets of $u$ and d quarks as well as that energy per baryon for strange quark matter must be below 930.4 MeV). For the MIT bag model we can find that the lowest rotational frequency at which triaxial instability can develop is $\sim 0.75 \mathrm{kHz}$.

\subsubsection{Lower limits on the maximum orbital frequency around rotating strange quark stars and kilohertz QPOs}

In this section we show that triaxial instability is important for interpretation of maximal orbital frequency of rapidly rotating strange stars in LMXBs. We focus on the MIT bag model but our results are robust for other linear self-bound EOS. The spinup of a relativistic star crucially depends on the maximum orbital frequency around it, as do a host of other high energy accretion phenomena in low mass X-ray binaries, including quasi-periodic oscillations (QPOs) in the X-ray flux. Kluźniak et al. (1990) suggested that the mass of a neutron star may be derived by observing the maximum orbital frequency, if it occurs in the marginally stable orbit, and that the low frequency QPOs occurring in X-ray pulsars will have their counterpart in LMXBs, at frequencies in the $\mathrm{kHz}$ range. Such $\mathrm{kHz}$ QPOs have indeed been discovered and their frequency used to derive mass values of about $2 M_{\odot}$, under the stated assumption (Kaaret et al. 1997; Zhang et al. 1998; Kluźniak 1998; Bulik 2000). In fact, the QPO frequency may correspond to a larger orbit, and hence a smaller mass (Kluźniak \& Abramowicz 2002). The question of whether quark stars may have maximum orbital frequencies as low as $1.07 \mathrm{kHz}$ in $4 \mathrm{U} 1820-30$ (reported by Zhang et al. 1998) has also been investigated (Bulik et al. 1999a,b; Stergioulas et al. 1999; Zdunik et al. 2000a,b; Gondek-Rosińska et al. 2001a,b). The main result of these considerations was that the lowest orbital frequency at the innermost marginally stable orbit (ISCO) is attained either for nonrotating massive configurations close to their maximum mass limit, or for configurations at the Keplerian mass-shedding limit. In order to obtain an orbital frequency in the ISCO as low as $1.07 \mathrm{kHz}$ for slowly rotating strange stars one has to consider a specific set of (rather extreme) MIT bag model parameters (Zdunik et al. 2000a,b) allowing gravitational masses higher than $2.2 M_{\odot}$. In contrast, for stars rotating close to the Keplerian limit, a low orbital frequency at the ISCO can be obtained for a broad range of stellar masses (Stergioulas et al. 1999; see Gondek-Rosińska et al. 2001a for discussion).

We present the dependence of the maximum orbital frequency (the frequency at the marginally stable orbit if it exists or the Keplerian frequency at the stellar surface, $f_{\text {orb,max }}=$ $f_{\text {orb }}\left(R_{*}\right)$ ) on the frequency of rotation $f$ for the set of constant baryon mass sequences of strange stars in Fig. 8. Marginally stable orbits exist for a broad range of masses and rotational rates (see e.g. Fig. 1 in Gondek-Rosińska \& Kluźniak 2002). As it turns out, the very low mass limit, represented in Fig. 8 by the case of $M_{\mathrm{b}}=0.01 M_{\odot}$, is described well by the Maclaurin spheroid for which analytic formulae for orbital frequencies have been derived and found to agree with the relativistic numerical models (Amsterdamski et al. 2002). For this sequence, an ISCO exists only for rotation rates larger than $\sim 950 \mathrm{~Hz}$. We indicate the onset of the (Newtonian) dynamical instability to non-axisymmetric perturbations by an asterisk. In the 


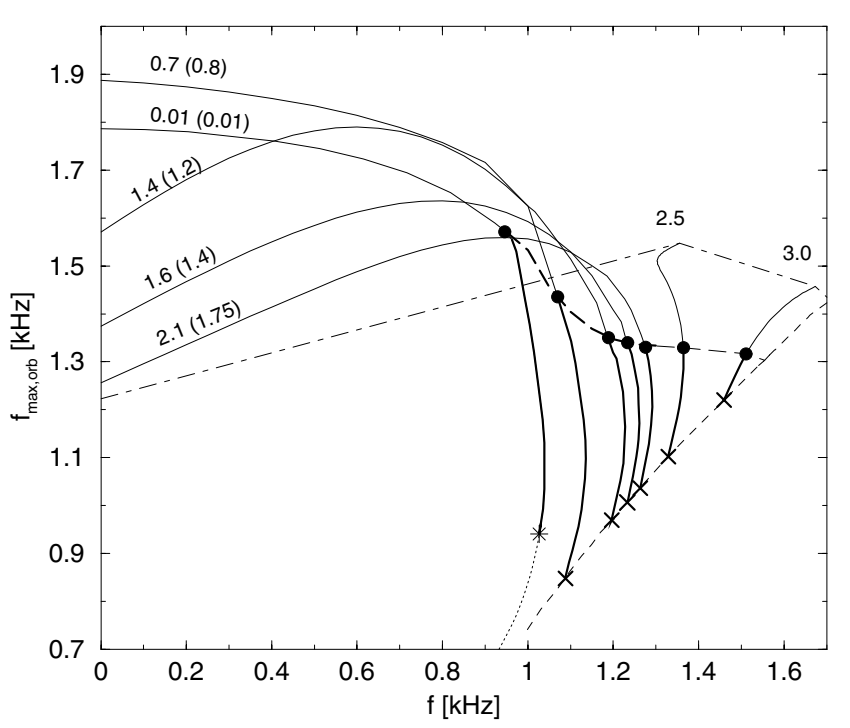

Fig. 8. The secularly unstable configurations to the bar mode formation (thick solid lines between a heavy black circle and a cross) on the maximum orbital frequency versus frequency of the rotation plane. The long dashed line indicates stars marginally stable to nonaxisymmetric perturbations taking into account viscosity as a leading dissipative mechanism. One sequence for a very low mass star (with $M_{\mathrm{b}}=0.01 M_{\odot}$ ) is shown, the critical point on this sequence for Newtonian dynamical instability to non-axisymmetric perturbations is indicated by an asterisk and dynamically unstable configurations are denoted with the dotted line.

Newtonian limit, the gap between the marginally stable orbit and the stellar surface is produced by the oblateness of the rapidly rotating low-mass quark star (Kluźniak et al. 2001; Amsterdamski et al. 2002; Zdunik \& Gourgoulhon 2001). We see that very rapidly rotating strange stars are secularly unstable to bar mode formation if the viscosity is high enough. The lowest frequency in the marginally stable orbit is obtained for slowly rotating massive strange stars or a marginally stable configuration to nonaxisymmetric perturbation (long dashed line). If the mass of the strange quark is relatively small, $m_{\mathrm{s}} c^{2} \sim 100 \mathrm{MeV}$, then the $r$-modes instability could play an important role as an emission mechanism of gravitational waves and a mechanism spinning down rotation rates of strange stars (Andersson et al. 2002).

\section{Rotating strange quark stars as sources of gravitational waves}

The interesting question is whether the viscosity-driven instability domain can be reached by any astrophysical process. In Sect. 5 we located the onset of instability (when the frequency of an $l=-m$ mode goes through zero in the corotating frame) along constant baryon sequences of rotating strange quark stars. This necessary condition yields the critical angular velocity for a given gravitational mass of a star. From our numerical results, the rotation frequency has to be above $0.8-1.2 \mathrm{kHz}$ for strange quark stars described by the MIT bag model of normal quark matter (any kind of superconductivity being excluded). The instability can develop for a broad range of gravitational masses of rotating SS.
The above results were obtained under the assumption of infinite shear viscosity. The necessary requirement is that the "real" viscosity has to be high enough to dissipate energy and let the instability develop along the Jacobi path.

As we mentioned in Sect. 2 if both viscosity and gravitational radiation are operative, they act against each other. Since the viscosity-driven mode is triaxial it tends to be damped by gravitational-wave emission, and since the gravitational-wave driven mode involves differential rotation, it is damped by viscosity. The instability can be important if the typical instability growth rate $\left(\tau_{\eta}\right.$ shear viscosity timescale) is faster than any damping mechanisms.

It is important to emphasize that the spin evolution of strange stars depends on many different physical mechanism, concerning e.g. viscosities of dense matter, cooling evolution, the influence of magnetic field on instabilities etc, for which our understanding is still unsatisfactory. Below we consider different timescales (shear viscosity, bulk viscosity, cooling timescale, gravitational radiation) and provide a crude estimate of the stellar temperature for which viscous effects could dominate. Viscosity driven instability is divergence free so bulk viscosity does not influence it. The shear and bulk viscosity (axial modes are divergence free but mode coupling should be taken into account) of strange quark star matter is able to suppress the growth of the CFS-instability except when the star passes through a certain temperature window. The $l=m=2 r$-mode is expected to lead to the strongest CFS-instability so we will take into account only this mode in our discussion.

\subsection{Viscous timescales connected to shear viscosity}

Shear viscosity of normal SQM has been calculated by Heiselberg \& Pethick (1993):

$\eta=5 \times 10^{15}\left(\frac{0.1}{\alpha}\right)\left(\frac{\rho}{\rho_{0}}\right)^{14 / 9} T_{9}{ }^{-5 / 3} \mathrm{~g} \mathrm{~cm}^{-1} \mathrm{~s}^{-1}$,

where $T_{9}=T / 10^{9} \mathrm{~K}$. Assuming $\rho=4 \rho_{0}, \alpha_{\mathrm{s}}=0.1$, and $R=$ $10 \mathrm{~km}$, we estimate the viscous timescale for a SS as

$\tau_{\eta}=\frac{\rho R^{2}}{\eta}=5.3 \times 10^{8} T_{9}^{5 / 3} \mathrm{~s}$.

This is characteristic timescale of the angular momentum transport within a strange star.

\subsection{Viscous timescales connected with bulk viscosity}

Bulk viscosity $\zeta$ of the SQM is due to the non-equilibrium nonleptonic strangeness changing process

$u+d \rightleftharpoons s+u$.

Local compression or decompression of the SQM drives it from the flavor equilibrium, because chemical potentials for the ultrarelativistic up and down quarks have weaker density dependence than the chemical potential of the massive strange quarks. Therefore, a baryon density perturbation implies $\mu_{\mathrm{s}} \neq$ $\mu_{\mathrm{d}}$. The non-equilibrium reactions, changing the concentrations of the down and strange quarks, and driving the system toward 
equilibrium, are irreversible, and therefore imply dissipation. The dissipation rate depends sensitively on the strange quark mass. Of course, dissipation vanishes in the (unphysical) limit of $m_{\mathrm{s}} \longrightarrow 0$, where the flavor symmetry is enforced. The dissipation rate in a pulsating SQM depends on the temperature, density, frequency of pulsations $\omega$, and on the density oscillation amplitude $\delta n_{\mathrm{b}}$. The time-averaged dissipation rate can be represented by the (effective) bulk viscosity of SQM. The formula for $\zeta$ was derived by Madsen (1992), and can be written as

$$
\begin{aligned}
\zeta= & 9 \times 10^{27}\left(\frac{m_{\mathrm{s}} c^{2}}{100 \mathrm{MeV}}\right)^{4}\left(\frac{\omega}{10^{4} \mathrm{~s}^{-1}}\right)^{-2} \frac{\rho}{\rho_{0}} T_{9}^{2} \\
& \times\left[1+b T_{9}{ }^{4}\left(\omega / 10^{4} \mathrm{~s}^{-1}\right)^{2}\right]^{-1},
\end{aligned}
$$

where the dimensionless coefficient $b$ is proportional to the coefficient $\beta$ of Madsen (1992), and the terms depending on $\delta n_{\mathrm{b}}$ were assumed to be unimportant (see also Andersson et al. 2002). The second term in square brackets is important for $T \gtrsim 10^{10} \mathrm{~K}$, where it leads to the quenching of $\zeta$, opening in this way the possibility of the $r$-mode instability in very hot rotating strange stars (Madsen 2000).

The terms depending on $\delta n_{\mathrm{b}}$ become important when

$$
\frac{\delta n_{\mathrm{b}}}{n_{\mathrm{b}}} \frac{m_{\mathrm{s}} c^{2}}{\mu_{d} T_{9}}>2
$$

Such a condition can be realized at low temperatures $T \lesssim 10^{8} \mathrm{~K}$ only provided $\delta n_{\mathrm{b}} / n_{\mathrm{b}}$ in the $r$-mode is sizable, $\delta n_{\mathrm{b}} / n_{\mathrm{b}} \gtrsim 0.1$ : this could happen only for a large-amplitude $r$-mode instability. In what follows, the second term in the square brackets will be omitted.

Consider now $T<5 \times 10^{9} \mathrm{~K}$, when the square bracket in Eq. (5) can be replaced by one. Using results of Madsen (2000) and assuming a mean density of strange star $3 \rho_{0}$, we get the following rough estimate for the viscous damping timescale of the $r$-mode,

$T<5 \times 10^{9} \mathrm{~K}:$

$\tau_{\zeta} \simeq 4\left(\frac{m_{\mathrm{s}} c^{2}}{100 \mathrm{MeV}}\right)^{-4}\left(\frac{f}{1000 \mathrm{~Hz}}\right)^{-2} T_{9}^{-2} \mathrm{~s}$.

The damping timescale depends strongly on $m_{\mathrm{s}}$, which is a renormalized strange quark mass (see Sect. 3.1).

\subsection{Cooling timescale}

The SS cools rapidly, with

$\tau_{\text {cool }} \equiv \frac{T}{\dot{T}}=\frac{c_{V} T}{Q_{v}}$

where $c_{V}$ is the specific heat of SQM (at a fixed volume), and $Q_{v}$ is the neutrino emissivity. Equation (8) can be easily integrated to give the time (age) needed to cool SS to $T_{8} \equiv T / 10^{8} \mathrm{~K}$,

$t_{\text {cool }}=\frac{10^{8}}{T_{8}^{4}} \mathrm{~s}$.

\subsection{Damping of the Jacobi-like instability}

Lindblom \& Detweiler (1977) have shown that if both viscosity and gravitational radiation reactions are taken into account, rotating stars can be stable beyond the Jacobi bifurcation point located in Sect. 5. This stability can be seen as resulting from a competition between the CFS Dedekind-like mode and the Jacobi-like mode. It is governed by the ratio (deduced from Eq. (8) of Lindblom \& Detweiler 1977)

$X=4.41 \times 10^{-13}\left(\frac{v}{\mathrm{~cm}^{2} \mathrm{~s}^{-1}}\right)\left(\frac{R}{10 \mathrm{~km}}\right)^{2}\left(\frac{M}{1.4 M_{\odot}}\right)^{3}$,

where $v=\eta / \rho$ is the kinematic shear viscosity. If $X \gg 1$ the star is unstable with respect to the Jacobi-like mode (viscositydriven instability), whereas if $X \ll 1$, it is unstable with respect to the Dedekind-like mode (CFS instability). In the intermediate regime, $X \sim 1$, the star is stable well beyond the bifurcation point. The formula (10) has been established for incompressible stars. Taking into account the compressibility does not change it significantly, since for polytropes of adiabatic index $\gamma$ this merely adds a factor $(\gamma-6 / 5)^{2} /(\gamma-1)^{2}$ in front of it (Lai \& Shapiro 1995).

Putting in Eq. (10) the value of $v$ deduced from the shear viscosity given by Eq. (2) leads to

$$
\begin{aligned}
X= & 2 \times 10^{-8}\left(\frac{0.1}{\alpha}\right)\left(\frac{\rho}{\rho_{0}}\right)^{5 / 9}\left(\frac{10^{9} \mathrm{~K}}{T}\right)^{5 / 3} \\
& \times\left(\frac{R}{10 \mathrm{~km}}\right)^{2}\left(\frac{M}{1.4 M_{\odot}}\right)^{3} .
\end{aligned}
$$

The fact that $X \ll 1$ for astrophysicaly interesting temperatures $T>10^{5} \mathrm{~K}$ (see Eq. (9)) means that the viscosity-driven instability cannot develop in rotating strange stars described by the MIT bag model, even if a star rotates rapidly with $T /|W|>(T /|W|)_{\text {crit }}$.

\subsection{The instability windows for $r$-modes}

The CFS-instability of $r$-modes in strange stars has been studied by Madsen (2000) and Andersson et al. (2002) in the Newtonian limit. They found the critical rotation frequency for a given stellar model as a function of temperature solving the following equation

$\frac{1}{\tau_{\mathrm{r}}}+\frac{1}{\tau_{\eta}}+\frac{1}{\tau_{\zeta}}=0$

where $\tau_{\mathrm{r}} \sim-22(47)\left(\frac{M}{1.4 M_{\odot}}\right)^{-1}\left(\frac{R}{10 \mathrm{~km}}\right)^{-4}\left(\frac{f}{\mathrm{kHz}}\right)^{-6} \mathrm{~s}$ (Kokkotas \& Stergioulas 1999) is the $r$-mode growth time-scale, taking into account only the leading current multipole and assuming a constant density profile (or $n=1$ polytrop). According to this study, the instability operates in a very different temperature window than for NS (see Fig. 1 in Madsen 2000 for the dependence of critical spin frequency on internal temperature for the MIT bag model strange star with $m_{\mathrm{s}} c^{2}=100$ and $200 \mathrm{MeV}$ ). Since for SS bulk viscosity becomes weak at both very low and very high temperatures, the $r$-mode instability could occur in 


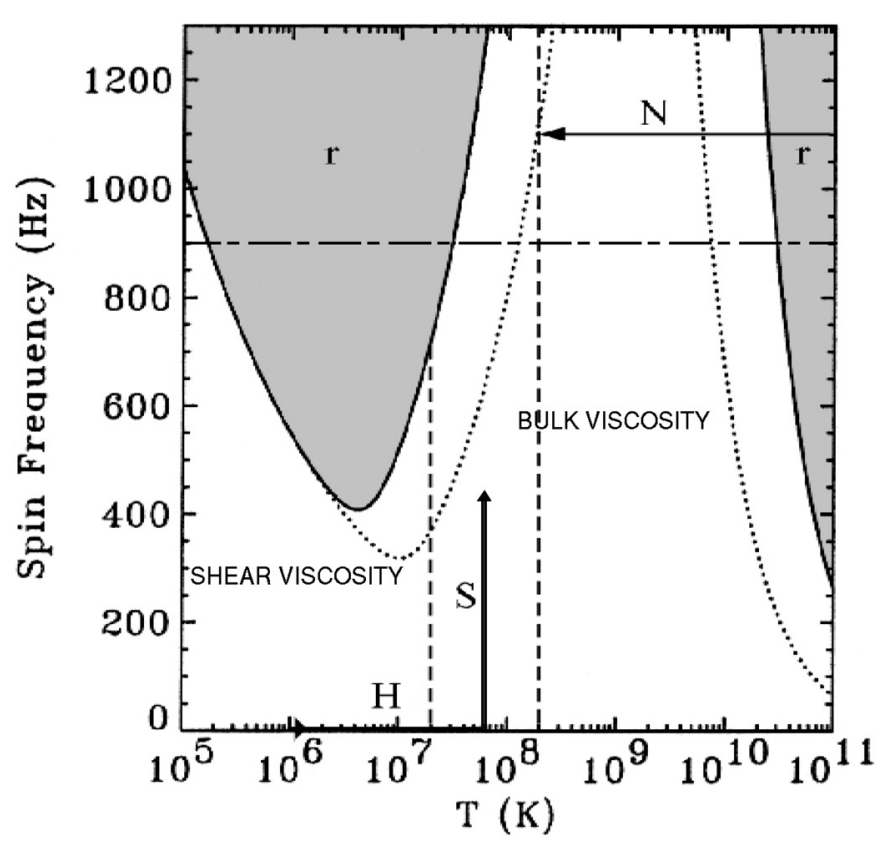

Fig. 9. Regions of the $r$-mode instability (hatched region with $r$-label) for a canonical mass strange star assuming $m_{\mathrm{s}} c^{2}=200 \mathrm{MeV}$ in the rotational frequency-temperature plane. The $r$-mode instability domain for $m_{\mathrm{s}} c^{2}=100 \mathrm{MeV}$ is shown for comparison (regions above dotted lines). The $r$-mode instability windows are taken from Madsen 2000. The short-long dashed horizontal line corresponds to the critical rotation rate for viscosity driven instability assuming canonical stellar mass, $m_{\mathrm{s}} c^{2}=200 \mathrm{MeV}$ and low MIT bag constant $40 \mathrm{MeV} / \mathrm{fm}^{3}$. The $\mathrm{H}-\mathrm{S}$ tract corresponds to the evolution of an old accreting SS and $\mathrm{N}$ tract to a newly born strange star.

temperature windows $T>10^{10} \mathrm{~K}\left(4 \times 10^{9} \mathrm{~K}\right)$ and $T<10^{7} \mathrm{~K}$ $\left(2 \times 10^{8} \mathrm{~K}\right)$ for $m_{\mathrm{s}} c^{2}=200 \mathrm{MeV}$, shown as the hatched area in our Fig. 9 (for $m_{\mathrm{s}} c^{2}=100 \mathrm{MeV}$ above dotted line), if the cooling time is long enough. Andersson et al. (2002) suggested that the $r$-modes in a strange star never grow to large amplitudes and emit a persistent gravitational wave signal.

\subsection{Astrophysical scenarios}

Let us consider two evolutionary scenarios for rotating strange stars. The first scenario refers to a newly born, rapidly rotating $\mathrm{SS}$, cooling due to neutrino emission. The second scenario is that of a SS in a close binary system, which heats and spins-up due to accretion of matter. In both cases we assume that strange quark matter (SQM) is normal (non-superconducting).

There are important differences between the two astrophysical scenarios mentioned above. The first one is that the energy radiated by a newborn relativistic star is supplied by its rotational kinetic energy, if it acquired a sufficiently large amount of rotational energy during its formation. For an accreting compact star it is supplied by the accreted matter. An old strange star in a close binary system, accreting matter from its companion, may be spun up until the ratio of $T /|W|$ is high enough to allow for the symmetry breaking. A steady state regime is achieved when the total accreted angular momentum is evacuated via gravitational radiation (Wagoner 1984; Papaloizou \& Pringle 1978), this process being known as forced gravitational emission or the Wagoner mechanism. In this case the frequency of the emitted gravitational waves depends upon the type of triaxial instability. For the main $l=m=2 r$-mode the frequency of the emerging gravitational waves is (see e.g. Stergioulas 1998)

$f_{\mathrm{gw}}^{r-\text { mode }}=\frac{4}{3} f$

For the Jacobi-like bar mode instability, the frequency of gravitational waves is simply twice the rotational frequency:

$f_{\mathrm{gw}}^{\mathrm{visc}}=2 f$.

If, in addition to the measure of $f_{\mathrm{gw}}$ by a gravitational wave detector, one knows $f$ from electromagnetic observations (e.g. $\mathrm{X}$-ray pulsations), one can determine the kind of secular rotational instabilities acting on the star, and consequently have some insight into the value of the physical parameters (viscosity, temperature) inside the compact object.

\subsubsection{Newly born rapidly rotating strange star}

A SS is born from a proto neutron star with an initial temperature of $\sim 10^{11} \mathrm{~K}$, and cools via neutrino emission (shown in a schematic way as the $\mathrm{N}$ track in Fig. 9). Of two basic types of SQM viscosity, the bulk one is crucial for stabilizing rapid rotation with respect to the $r$-mode instability (which develops on a timescale $\tau_{\mathrm{r}}$ ). The region of the $r$-mode instability in the $f-T$ plane strongly depends on the value of $m_{\mathrm{s}}$, because $\tau_{\zeta} \propto m_{\mathrm{s}}^{-4}$. Had we taken $m_{\mathrm{s}} c^{2}=100 \mathrm{MeV}$, the $r$-mode instability of newly born SS would extend to much lower $T$ and $f$. We use renormalized s quark mass $m_{\mathrm{s}} c^{2}=200 \mathrm{MeV}$ consistent with particle data tables (see Sect. 3.1). Then the $r$-mode is unstable within the right hatched strip in Fig. 9. Due to the strong temperature dependence of $\tau_{\zeta}$, stability of rotation is regained as soon as $T$ decreases below $10^{10} \mathrm{~K}$, which takes only a few seconds (see Eq. (9)). A rapidly cooling young hot strange star would not be spun down significantly passing the right $r$-mode instability window.

At $T=10^{8} \mathrm{~K}, \tau_{\eta} \sim 10^{7} \mathrm{~s} \ll t_{\text {cool }}\left(10^{8} \mathrm{~K}\right)=10^{8} \mathrm{~s}$ and a star could rotate rigidly. Since $X<<1$ (due to a very short gravitational damping time-scale) the viscosity driven instability cannot develop even if the newly born SS left the $r$-mode instability strip with $T /|W|>(T /|W|)_{\text {crit }}$.

Several years after its birth a star will reach the low- $T$ (left) (when temperature is a few times $10^{7}-10^{8} \mathrm{~K}$ ) $r$-mode instability strip. During its cooling it will lose the excess of angular momentum via GW radiation until it leaves the low- $T r$-mode instability window.

\subsubsection{Spin evolution of an old accreting strange star}

Consider an old SS which starts to accrete matter in a close binary system. Its initial internal temperature was very low, but energy release associated with accretion will induce heating of SS interior. To be specific, we consider accretion of a hydrogen-rich plasma. There are two main channels of heating by accretion. The first one is connected to plasma falling onto a bar SS surface, and the second one involves formation 
of the crust and thermonuclear burning of compressed plasma (Haensel \& Zdunik 1991). In both cases, additional heat release occurs at the SQM core edge, where absorption and deconfinement of protons is associated with exothermic irreversible reactions $p \longrightarrow 2 u+d, u+d \longrightarrow s+u$. The energy release per one deconfined proton is $q_{\mathrm{s}}$. In what follows we will assume $q_{\mathrm{s}}=50 \mathrm{MeV}$. After some $10^{5} \mathrm{yrs} \mathrm{SS}$, accreting matter at a constant rate $\dot{M}$, it will reach the steady thermal state, in which heating due to accretion and deconfinement is balanced with neutrino losses from the SS core (see Fujimoto et al. 1984). Notice that at a fixed accretion rate (which will be expressed in the units of $10^{-8} M_{\odot} \mathrm{yr}^{-1}$ ), $\dot{M}_{-8}$, reaching of the steady state corresponds to accretion of only $10^{-3} \dot{M}_{-8} M_{\odot}$ (H-track in Fig. 9). Equating heat gains and losses, one can estimate internal temperature of accreting SS (Haensel \& Zdunik 1991),

$T=1.1 \times 10^{8}\left(\xi M_{*} \dot{M}_{-8}\right)^{1 / 6} R_{6}^{-2 / 3} \mathrm{~K}$,

where $M_{*}=M / M_{\odot}$ and $R_{6}=R / 10^{6} \mathrm{~cm}$. The parameter $\xi$ varies from $\xi=0.2$ in the limiting case when the heating results exclusively from the deconfinement process, to $\xi=1.2$ when all gravitational energy release by infalling protons flows into the SS core (Haensel \& Zdunik 1991). Putting $M_{*}=1.6$ and $R_{6}=1, \xi=0.2$, we get $T=0.9 \times 10^{8} \mathrm{~K}$.

Further accretion of some $(0.3-0.4) \quad M_{\odot}$ during some $5 \times 10^{7}$ yrs at $\dot{M}_{-8}=1$ could spin-up the SS to frequency $f \sim 1 \mathrm{kHz}$, at a nearly constant temperature (Zdunik et al. 2002) (the vertical S-track in Fig. 9). Along the S-track, for $m_{\mathrm{S}}=200 \mathrm{MeV}$, a star is stable with respect to the $r$-mode instability because of its huge bulk viscosity. It can be accelerated to high rotational frequency reaching the critical frequency for viscosity driven instability (short-long dashed line in Fig. 9). After crossing $f=f_{\text {crit }}=(0.9-1.2) \mathrm{kHz}$ (the precise value depending on the SS mass and the detailed form of the SQM EOS) the SS is stable to the bar mode formation since the characteristic growth time-scale of viscosity driven instability $\tau_{\zeta} \sim$ a month $>>$ than tens of seconds - the gravitational wave damping time. In the case of $m_{\mathrm{s}}=200 \mathrm{MeV}$ a strange star can be accelerated to very high rotational frequencies until it reaches one of the upper limits (low- $T r$-mode instability region, dynamical instability limit, mass-shedding limit).

Let us stress the importance of the value of $m_{\mathrm{s}}$ for such an astrophysical scenario. For high mass of strange quark $>200 \mathrm{MeV}$ a star can be accelerated to very high frequencies without emitting gravitational waves. If on the contrary the strange quark mass is $m_{\mathrm{s}} \leq 100 \mathrm{MeV}$ the low- $T r$ mode instability window (above the dotted line in Fig. 9), it can be reached at a rotation rate of $0.4-0.6 \mathrm{kHz}$. The rotating $\mathrm{SS}$ is expected to be in a quasi-stationary state, in which the angular momentum losses via gravitational radiation are balanced by the accretion torque. In this steady state an accreting SS is an emitter of a persistent gravitational wave signal.

\subsubsection{The role of the magnetic field}

Our results could be changed if we take the magnetic field into account. The presence of a strong magnetic field in SQM could also contribute to the angular momentum transport and therefore influence different kinds of gravitational instabilities. The electrical conductivity of the SQM is huge. Using the formula derived by Heiselberg \& Pethick (1993) we get $\sigma \simeq(0.1 / \alpha)^{5 / 3}\left(\rho / \rho_{0}\right)^{8 / 9} T_{9}^{-5 / 3} 10^{21} \mathrm{~s}^{-1}$. Therefore, the magnetic field is frozen in SQM which combined with the presence of rapid and differential rotation could lead to additional viscous mechanisms due to the magnetorotational instability.

Although a high conductivity is a key element of compact stars (neutron stars, strange stars) physics there have been only a few discussions of the effect of magnetic fields on triaxial instabilities (Bonazzola et al. 1996; Spruit 1999; Rezzolla et al. 2000; Ho \& Lai 2000). It was suggested by Bonazzola et al. (1996) that the magnetic field can prevent the Dedekind-like mode. Assuming infinite conductivity, shear of the fluid from differential angular velocity generates some toroidal magnetic field in addition to the poloidal one (Bonazzola \& Marck 1994). The excess of kinetic energy contained in differential rotation with respect to rigid rotation can be efficiently converted into magnetic energy, enforcing rigid rotation.

Ho \& Lai (2000) have studied the combined effects of magnetic braking and gravitational radiation generated by $r$-mode instability on the spin evolution of young rapidly rotating neutron stars. They suggested that a strong magnetic field can drive the instability just like gravitational radiation reaction. The question of whether an unstable $r$-mode leads to differential rotation, and whether the mode can be prevented from growing by the magnetic field, was discussed by Rezzolla et al. (2000). They studied how so-called Stokes drift affects the magnetic field of the star, and what the backreaction on the mode may be. It was estimated that the instability could operate in young neutron stars and recycled ones provided that they spin fast enough.

\section{Summary}

We have performed numerical investigations of the Jacobi-like bar mode secular instability of rotating strange quark stars described by the MIT bag model. In particular, we have computed the domain of the parameter space of stationary rigidly rotating configurations in which triaxial instability develops. It has been found that, contrary to neutron stars, the triaxial instability can set in quite far towards the mass-shed limit and is allowed for small mass stars as well if the viscosity is high enough to damp out any deviation from rigid rotation. The lower mass is the lower rotational frequency at which Jacobilike instability can develop. The viscosity driven instability does not set an upper limit on the spin rate (but on $T /|W|$ or oblateness) of strange stars - the configuration marginally stable against non-axisymmetric perturbation can rotate with a frequency higher than mass-shedding configurations (the maximal rotating configuration is not a Keplerian one for SS). A normal and low mass supramassive strange star gaining angular momentum always slows down before reaching the Keplerian limit. For a high-mass supramassive strange star sequence, the mass-shedding configuration is the one with the lowest rotational frequency in the sequence. The instability, if relevant, can impose a strong constrain on the lower limit of the frequency at the innermost stable circular orbit around rapidly rotating strange stars. The results are robust for all linear selfbound equations of state. The critical rotation frequency for 
viscosity driven instability in the case of strange stars described by the MIT bag model is high, $0.8-1.2 \mathrm{kHz}$ (depending on the stellar mass and the detailed form of the SQM EOS).

We have discussed astrophysical scenarios when triaxial instabilities ( $r$-mode and viscosity driven instability) could be relevant in strange stars described by the standard MIT bag model of normal quark matter (any kind of superconductivity being excluded). We have shown, taking into account actual values of shear viscosity, that viscosity driven instability cannot develop in any astrophysicaly relevant temperature windows.

Whether a strange star can be a good emitter of gravitational waves or not depends on CFS instability. In our discussion we focused on the most promising CFS instability ( $r$ mode). The spin evolution of strange stars and emission of gravitational waves strongly depend on the mass of the strange quark (caused by the strong dependence of bulk viscosity on the mass of a strange quark). If we take the strange quark mass to be $m_{\mathrm{s}} c^{2} \sim 200 \mathrm{MeV}$ or higher (consistent with renormalization and particle data tables) then $r$-mode instability is relevant only in a narrow temperature window and seems to be unimportant in Low Mass X-Ray Binaries. In this case an old star can be spun up in LMXBs to very high rotation rates and a low frequency at the marginally stable orbit can be obtained $(\sim 1 \mathrm{kHz})$.

If on the contrary the strange quark mass is low, $m_{\mathrm{S}} c^{2} \sim$ $100 \mathrm{MeV}$, then a star accelerated in LMXBs to a frequency of $0.4-0.6 \mathrm{kHz}$ can be a good emitter of persistent gravitational waves (see Andersson et al. 2002 for a discussion) that ought to be detectable with large-scale interferometers. In this case the $r$-mode instability can impose strong constraints on the lower limits on rotational period and then on the maximal orbital frequency in LMXBs. The lowest frequency at the marginally stable orbit could be obtained for slowly-rotating massive strange stars.

A young strange star would not be spun down significantly by the $r$-modes during its first few months of existence (because of its very rapid cooling rate) until the star has cooled to a temperature of a few times $10^{7}-10^{8} \mathrm{~K}$.

The astrophysical scenario will change if values of viscosity change or/and different physical mechanisms e.g. magnetic field will be taken into account.

Acknowledgements. We are grateful to N. Stergioulas, N. Andersson, L. Villain, S. Bonazzola, D. Lai, J. Friedman and K. Kokkotas for helpful discussions. This work has been funded by the following grants: KBN grants 5P03D.017.21 and 5P03D.020.20; the Greek-Polish Joint Research and Technology Programme EPANM.43/2013555 and the EU Programme "Improving the Human Research Potential and the Socio-Economic Knowledge Base" (Research Training Network Contract HPRN-CT-2000-00137).

\section{References}

Alcock, C., Farhi, E., \& Olinto, A. 1986, ApJ, 310, 261

Amsterdamski, P., Bulik, T., Gondek-Rosińska, D., \& Kluźniak, W. 2002, A\&A, 381, L21

Andersson, N. 2003, Class. Quantum Grav., 20, R105

Andersson, N., Jones, D. I., \& Kokkotas, K. D. 2002, MNRAS, 337, 2124
Andersson, N., \& Kokkotas, K. D. 2001, Int. J. Mod. Phys. D, 10, 381

Bonazzola, S., Frieben, J., \& Gourgoulhon, E. 1996, ApJ, 460, 379

Bonazzola, S., Frieben, J., \& Gourgoulhon, E. 1998b, A\&A, 331, 280

Bonazzola, S., Gourgoulhon, E., \& Marck, J. A. 1998a, Phys. Rev. D., 58,104020

Bonazzola, S., \& Marck, J.-A. 1994, Ann. Rev. Nucl. Part. Sci., 45, 655

Bulik, T., Gondek-Rosińska, D., \& Kluźniak, W. 1999, A\&A, 344, L71

Bulik, T., Gondek-Rosińska, D., \& Kluźniak, W. 1999, Astrophys. Lett. Comm., 38, 77

Chandrasekhar, S. 1969, Ellipsoidal figures of equilibrium (New Haven: Yale University Press)

Cook, G. B., Shapiro, S. L., \& Teukolsky, S. A. 1994, ApJ, 424, 823

Dey, M., Bombaci, I., Dey, J., Ray, S., \& Samanta, B. C. 1998, Phys. Lett. B, 438, 123

Di Girolamo, T., \& Vietri, M. 2002, ApJ, 581, 519

Drake, J. J., Marshall, H. L., Dreizler. S., et al. 2002, ApJ, 572, 996

Farhi, E., \& Jaffe, R. L. 1984, Phys. Rev. D, 30, 2379

Friedman, J. L. 1978, Commun. Math. Phys., 62, 247

Friedman, J. L., Ipser, J. R., \& Parker, L. R. 1986, ApJ, 304, 115; errata published in ApJ, 351, 705 (1990)

Friedman, J. L., Ipser, J. R., \& Sorkin, R. D. 1988, ApJ, 325, 722

Friedman, J. L., \& Lockitch, K. H. 2002, in Proc. Of the 9th Marcel Grossmann meeting, ed. V. G. Gurzadyan, R. T. Jantzen, R. Ruffini (Singapore: World Scientific)

Friedman, J. L., \& Shutz, B. F. 1978, ApJ, 222, 281

Fujimoto, M. Y., Hanawa, T., \& Miyaji, S. 1981, ApJ, 247, 267

Gondek-Rosińska, D., Haensel, P., Zdunik, J. L., \& Gourgoulhon, E. 2000a, Astron. Soc. Pac. Conf. Ser., 202, 661 [astro-ph/0009282]

Gondek-Rosińska, D., Bulik, T., Zdunik, J. L., et al. 2000b, A\&A, 363,1005

Gondek-Rosińska, D., Stergioulas, N., Bulik, T., Kluźniak, W., \& Gourgoulhon, E. 2001a, A\&A, 380, 190

Gondek-Rosińska, D., Bulik, T., Kluźniak, W., Zdunik, J. L., \& Gourgoulhon, E. 2001b, Exploring the gamma-ray universe. Proceedings of the Fourth INTEGRAL Workshop, 4-8 September 2000, Alicante, Spain. Editor: B. Battrick, Scientific editors: A. Gimenez, V. Reglero \& C. Winkler. ESA SP-459, Noordwijk: ESA Publications Division, ISBN 92-9092-677-5, 223 [astro-ph/0012540]

Gondek-Rosińska, D., \& Gourgoulhon, E. 2002, Phys. Rev. D., 66, 044021

Gondek-Rosińska, D., \& Kluźniak, W. 2002, The proceedings of the XXXVIIth Rencontres de Moriond, XIInd Moriond Astrophysics Meeting, The Gamma-Ray Universe, Les Arcs, Savoie, France, March 9-16, 2002, Series: Moriond Astrophysics Meetings, Edited by A. Goldwurm, D. N. Neumann, J. Tran Thanh Van, 517 [astro-ph/0206363]

Gondek-Rosińska, D., Bulik, T., Zdunik, J. L., et al. 2004, in preparation

Gourgoulhon, E., Haensel, P., Livine, R., et al. 1999, A\&A, 349, 851

Groom, D. E., et al. (Particle Data Group) 2000, Eur. Phys. J., C15, 1

Haensel, P., Zdunik, J. L., \& Schaeffer, R. 1986, A\&A, 160, 121

Haensel, P., \& Zdunik, J. L. 1991, Nuc. Phys. B (Proc. Suppl.), 24B, 139

Heiselberg, H., \& Pethick, C. J. 1993, Phys. Rev. D, 48, 2916

Ho, W. C. G., \& Lai, D. 2000, ApJ, 543, 386

Kaaret, P., Ford, E. C., \& Chen, K. 1997, ApJ, 480, 127

Kluźniak, W. 1998, ApJ, 509, L37

Kluźniak, W., Bulik, T., \& Gondek-Rosińska, D. 2000, ESA-SP 459, 301 [astro-ph/0011517] 
Kluźniak, W., Michelson, P., \& Wagoner, R. V. 1990, ApJ, 358, 538

Kluźniak, W., \& Wagoner, R. V. 1985, ApJ, 297, 548

Kokkotas, K. D., \& Stergioulas, N. 1999, A\&A, 341, 110

Komatsu, H., Eriguchi, Y., \& Hachisu, I. 1989, MNRAS, 237, 355

Lai, D., \& Shapiro, S. L. 1995, ApJ, 442, 259

Lindblom, L., \& Detweiler, S. L. 1977, ApJ, 211, 565

Madsen, J. 1992, Phys. Rev. D, 46, 3920

Madsen, J. 2000, Phys. Rev. Lett., 85, 10

Miller, M. C., Lamb, F. K., \& Cook, G. B. 1998, ApJ, 509, 793

Morsink, S. M., Stergioulas, N., \& Blattnig, S. R. 1999, ApJ, 510, 854

Nozawa, T., Stergioulas, N., Gourgoulhon, E., \& Eriguchi, Y. 1998, A\&AS, 132, 431

Papaloizou, J., \& Pringle, J. E. 1978, MNRAS, 184, 501

Rezzolla, L., Lamb, F. K., \& Shapiro, S. L. 2000, ApJ, 531L, 139

Roberts, P. H., \& Stewartson, K. 1963, ApJ, 137, 777

Saijo, M., Shibata, M., Baumgarte, T. W., \& Shapiro, S. L. 2001, ApJ, 548,919

Shapiro, S. L., \& Teukolsky, S. A. 1983, Black holes, white dwarfs, and neutron stars (New York: Wiley)

Shapiro, S. L., \& Zane, S. 1998, ApJS, 117, 531

Shibata, M., Baumgarte, T. W., \& Shapiro, S. L. 2000, ApJ, 542, 453

Shibata, M., \& Sasaki, M. 1999, Phys. Rev. D, 60, 084002

Sibgatulin, N. R., \& Sunyaev, R. A. 1998, Astron. Lett., 24, 774
Stergioulas, N. 2003, Living Rev. Relativity, 6, 3

Stergioulas, N. 1998, Living Rev. Relativity, 1, 8 http://www. livingreviews.org/Articles/Volume1/ 1998-8stergio/

Stergioulas, N., \& Friedman, J. L. 1995, ApJ, 444, 306

Stergioulas, N., Kluźniak, W., \& Bulik, T. 1999, A\&A, 352, L116

Wagoner, R. V. 1984, ApJ, 278, 345

Wagoner, R. V., Silbergleit, A. S., \& Ortega-Rodriguez, M. 2001, ApJ, 559, L25

Walter, F. M., \& Lattimer, J. 2002, ApJ, 576, L145

Weber, F. 1999, Pulsars as Astrophysical Laboratories for Nuclear and Particle Physics (Bristol: IOP Publishing)

Witten, E. 1984, Phys. Rev., 30, 272

Zdunik, J. L. 2000, A\&A, 359, 311

Zdunik, J. L., Bulik, T., Kluźniak, W., Haensel, P., \& Gondek-Rosińska, D. 2000a, A\&A, 359, 143

Zdunik, J. L., Haensel, P., Gondek-Rosińska, D., \& Gourgoulhon, E. 2000b, A\&A, 356, 612

Zdunik, J. L., \& Gourgoulhon, E. 2001, Phys. Rev. D, 63, 087501

Zdunik, J. L., Haensel, P., \& Gourgoulhon, E. 2001, A\&A, 372, 535

Zdunik, J. L., Haensel, P., \& Gourgoulhon, E. 2002, A\&A, 381, 933

Zhang, W., Smale, A. P., Strohmayer, T. E., \& Swank, J. H. 1998, ApJ, 500, L171 